\title{
Combining Volumetric Soft Tissue Cuts for Interventional Surgery Simulation
}

\author{
Megumi Nakao ${ }^{1}$, Tomohiro Kuroda ${ }^{2}$, Hiroshi Oyama ${ }^{2}$, Masaru Komori ${ }^{3}$ \\ Tetsuya Matsuda ${ }^{1}$, and Takashi Takahashi ${ }^{2}$ \\ ${ }^{1}$ Graduate School of Informatics, Kyoto University, \\ Yoshida, Sakyo, Kyoto, 606-8501, Japan \\ meg@kuhp.kyoto-u.ac.jp \\ ${ }^{2}$ Department of Medical Informatics, Kyoto University Hospital \\ Shogoin, Sakyo, Kyoto, 606-8507, Japan \\ ${ }^{3}$ Computational Biomedicine, Shiga University of Medical Science, \\ Seta Tsukinowa, Otsu, 520-2192, Japan
}

\begin{abstract}
This paper proposes a framework to simulate soft tissue cuts for interventional surgery simulation. A strained status of soft tissues is modeled as internal tension between adjacent vertices in a particle based model. Both remodeling particle systems and an adaptive scheme in tetrahedral subdivision provide volumetric and smooth cuts on large virtual objects. 3D MRI datasets are applied to a developed system with a force feedback device. Measurement of the calculation time and visualization of simulation quality confirms that the framework contributes to surgical planning and training with tissue cutting.
\end{abstract}

\section{Introduction}

Minimally invasive surgery (MIS) is focused in the medical field, because small incision contributes to patient's beauty and risk reduction. Although MIS benefits patients, surgeons have to learn high procedural skills and have to experience more surgeries. Results of the latest surgeries, such as minimally invasive cardiac surgery (MICS), mostly depend on how to settle their surgical fields or local views. However, surgeons are currently forced to make decisions on surgical strategies empirically or intuitively using 2D or 3D images. For these issues, an advanced virtual reality based simulation has possibilities of giving a solution to both preoperative planning and training in surgical intervention. The planning system presented by Pflesser B. et al is a remarkable example in brain surgery [1]. The KISMET surgery simulator [2] gives an effective environment to learn surgical procedures in MIS.

This study aims to construct an advanced system with soft tissue cutting, which helps surgeons to discuss or learn optimum incision (cutting point and length), surgical path (direction and approach) and surgical field (view and space) in MICS [3]. However, providing accurate soft tissue cuts is one of challenging studies. The cutting model must handle topological change, biomechanical deformation, collision detection and haptic rendering. To support users with visual and haptic feedback, fast computational schemes are also important. So far, several approaches have been reported. Cutting methods applied to polygons are not effective to represent deep cut surface where inner tissues spread apart [4]. Voxel-based models [1] provide volumetric dis- 
play, whereas real time deformation of soft tissues is not achieved due to their large calculation cost. Although cutting frameworks based on a tetrahedral subdivision scheme [5] simulate volumetric cuts, there are still some problems in physical consistency, shape of cuts, and global manipulation on large dataset. In this scope, further development of advanced frameworks is mandatory to provide surgical realism in real time.

This paper presents an advanced framework that simulates interactive soft tissue cutting in surgical intervention. A strained status of soft tissues is modeled as internal tension between adjacent vertices in a particle based model. Both remodeling particle systems and an adaptive scheme in tetrahedral subdivision provide volumetric and smooth cuts on large virtual objects. In the following sections, details of the methods are described.

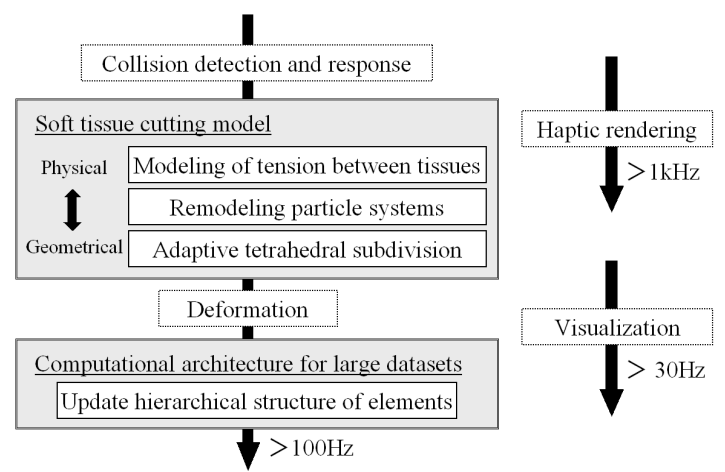

Fig. 1. A simulation framework for soft tissue cutting

\section{A Framework for Soft Tissue Cutting}

The proposed framework not only deals with large dataset (more than 10000 elements) but also aims to perform both topological change and real time deformation via global manipulation. In order to provide an interactive update rate, particle based models $[6,7]$ are currently our modeling solution capable of adequate accuracy and performance in physical simulation. Fig. 1 shows the developed framework that provides soft tissue cuts. The system has parallel algorithm loops for visual and haptic display. The visual loop is kept at least $100 \mathrm{~Hz}$ due to stability of the deformable model and real time rendering. A refresh rate of the haptic loop is $1000 \mathrm{~Hz}$ in order to satisfy force perception of the human.

The presented cutting model is a kind of package and can be integrated to foregoing physics based simulation. The computational architecture using an element hierarchical structure performs interactive and stable simulation for large datasets.

\section{Physical Balance and Transition of Soft Tissues}

The cutting model consists of three basic methods: modeling of internal tension, particle system remodeling and adaptive tetrahedral subdivision. 


\subsection{Modeling of Tension between Tissues}

Cutting simulation must deal with physical and geometrical models, and the algorithms have to be applied to virtual objects reconstructed from volumetric dataset of patients. From the viewpoint of consistent physical approaches, the authors focus on internal tension between soft tissues. Actually, all tension is in equilibrium at the ordinary state, and physical balance changes into the next stable state after incision. Although such physical behavior is essential to achieve simulation accuracy, it is not visible in the results of foregoing studies $[5,6]$. In a particle system, an initial state that internal tension is acting on tissues is represented by setting initial length of an edge shorter than initial distance between its two vertices. Using a tense parameter, initial length of an edge is determined by the following simple equation:

$$
l_{n}=d_{n}\left(1-s_{n}\right), 0 \leq s_{n} \leq 1
$$

where on an edge $n, l_{n}$ is suggested length, $d_{n}$ is initial distance between two vertices that the edge belongs to, and $s_{n}$ is a tense parameter. Although soft tissues of human bodies or organs have heterogeneous tension, the tense coefficient $s_{n}$ simulates realistic physical balance between in-vivo tissues.

\subsection{Physically Based Remodeling of Particle Systems}

This section describes remodeling particle systems in order to represent relaxation of soft tissues. Fig. 2 (a) shows a basic mass-spring system, which does not include damper elements in brief. In the proposed model, a pair of new vertices is created at the point clipped by a virtual scalpel, and then the system is modified on the condition that the system has the same behavior towards neighboring vertices like Fig. 2 (b).

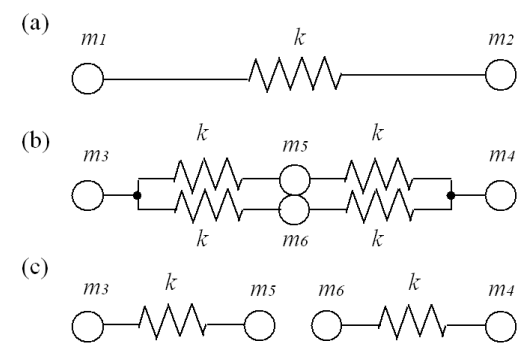

Fig. 2. Remodeling the particle system: initial tense state (a), creation and distribution of new vertices (b) and physical transition after elimination of alternative edges (c)

Mass of the two vertices is distributed to the new vertices. Then, we can cut into the system by elimination of alternative spring and damper elements connected to different vertices. For example in Fig. 2 (b), both edge $m_{3}-m_{5}$ and edge $m_{4}-m_{6}$ are eliminated. If the system has internal tension presented in the previous section, a pair of vertices physically spreads apart to new stable positions (Fig. 1 (c)). Movement of the vertices represents dynamic spread that provides interactive display. The mass of 
each vertex, which is proportional to volume of the elements it belongs to, is determined by the equation:

$$
m_{i}=!_{k \in E(i)} \frac{1}{4} \rho_{k} V_{k}
$$

where $m_{i}$ is mass of a vertex $i, E(i)$ is a set of elements that vertex $i$ belongs to, $\rho_{k}$ is density and $V_{k}$ is volume of an element $k$. The mass of the created vertices is also calculated by the equation (2).

\section{Adaptive Subdivision of Tetrahedral Objects}

According to the internal tension and elimination of spring/damper terms, physically based incision is represented using particle systems. However, we still have another problem as to topological transition and definition of a cut surface in order to display volumetric cuts. To solve this subject, the authors describe topological change of $3 \mathrm{D}$ meshes based on tetrahedral subdivision.

The generalized subdivision divides a tetrahedron into 17 smaller tetrahedra. One vertex is inserted at each edge and surface, and consequently 10 new vertices are created. However, radical increase of elements is a serious problem to keep the update rate stable and small. In order to achieve an interactive refresh rate, D. Bielser et al have defined a cut surface by inserting between three and five vertices [5]. A tetrahedron is subdivided into between four or six tetrahedra. In the next step, this paper gives adaptive tetrahedral subdivision that only creates three or four vertices and yields four or six minimal tetrahedra. Moreover, the adaptive scheme concerns accurate shape of cuts as well as consistent topological changes based on the concept of edge-plane intersection and movement of vertices. The implementation of the proposed method is simple because complex description of subdivision patterns is not required.

\subsection{Topological Change}

Intersection between a plane and a tetrahedron is described as two patterns in Fig. 3 . The intersection at four edges, shown in Fig. 3 (a), separates four vertices into a pair of two vertices, and the shape of the cross section is a square. The intersection at three edges, shown in Fig. 3 (b), separates four vertices into one vertex and three vertices, and the shape of the cross section is a triangle. This intersection gives a basic concept of creating a cut surface.

During cutting manipulation, movement of a virtual scalpel forms a clipping plane $C$, which clips a cut surface $S$ from a tetrahedral object $T$. Fig. 4 illustrates a relationship between the elements. Because $C$ is a partial plane, incomplete intersection is occurred at some tetrahedra. Therefore, two patterns of intersection in Fig. 3 cannot represent an adequate cut surface. This incomplete intersection is shown at the right tetrahedron of $T$ in Fig. 4.

In order to remove incomplete intersection, on the clipping plane $C$, adjacent vertices outside of the region $C$ are moved and replaced on its boundary $B$. A movement vector of the vertex is determined by average of edge vectors that intersect at the boundary $B$. A solution for incomplete intersection by vertex movement is illustrated in Fig. 5. 
(a)

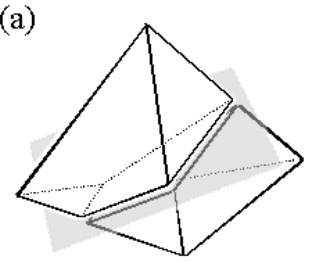

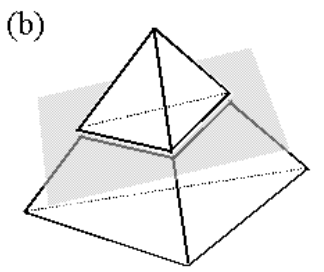

(b)

Fig. 3. Intersection between a plane and a tetrahedron

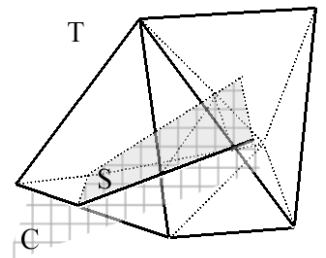

Fig. 4. Intersection between a clipping plane and tetrahedra ( $C$ : clipping plane, $S$ : cut surface, $T$ : tetrahedral object)

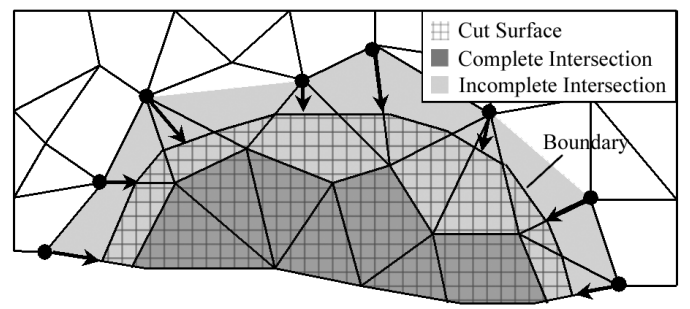

Fig. 5. Vertex movement and removal of incomplete intersection on the clipping plane

After movement of the vertices, incomplete intersection between $C$ and $T$ is removed. Consequently, the cut surface $S$ is defined completely by the two patterns of intersection in Fig. 3. The generated cut surface $S$ displays volumetric cut. The remodeling scheme of particle systems described in the section 3.2 is applied to all edges that intersect at $C$. The length of all edges that are affected by vertex movement is also recalculated.

\subsection{Topology Optimization}

Since a created vertex is currently connected to only one spring term, some edges are inserted into the object in order to simulate realistic cuts. Minimal tetrahedral subdivision in Fig. 6 gives consistent and effective patterns of edge insertion. In the case of four edges intersection, six new tetrahedra are generated like in Fig. 6 (a), and the intersection at three edges yields four new tetrahedra in Fig. 6 (b). Although some subdivision patterns can be described, an effective pattern carefully must be selected in order to achieve fine cuts. 
(a)

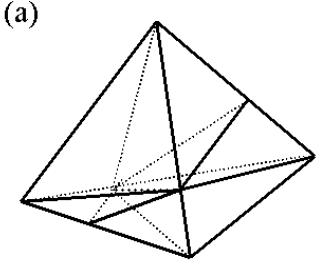

(b)

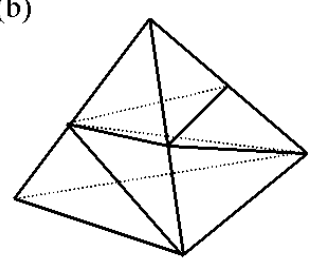

Fig. 6. Minimal tetrahedral subdivision

In the field of cutting simulation using physical models, improving shape of cuts is one of significant issues. So far, related works have reported that zigzag cut is a key problem for realistic surgery simulation [8]. In order to provide accurate and smooth cuts based on minimal tetrahedral subdivision, the authors provide an adaptive scheme, which selects the subdivision pattern that maximizes the minimum dihedral angle and minimizes the maximum dihedral angle. An indicator $\mu$ of each subdivision pattern is solved at the following equation:

$$
\mu=!_{i=0}^{N} \frac{\max \left(\theta_{i}(j, k)\right)}{\min \left(\theta_{i}(j, k)\right)}
$$

where four or six new tetrahedrons which are subdivided from a tetrahedron are described as $T_{i}(i=0, \ldots, N)$, and four faces of a new tetrahedron are described as $P_{i}(j)$ $(j=0, \ldots, 3)$. At this time, $\theta_{i}(j, k)$ is a dihedral angle between $P_{i}(j)$ and $P_{i}(k)$. If $\mu$ is small, we can estimate that the subdivision pattern has good quality.

\section{Results}

The overall algorithms of proposed methods are implemented under a standard PC (CPU: Pentium III Dual 933MHz, Memory: 2GB, and OS: Windows2000). A PHANToM (Sensable Technologies) is applied to a simulation system with a force feedback device. When a user manipulates a virtual scalpel and cuts into virtual objects, the system displays simulated soft tissue cuts and returns effective force feedback. To simulate interaction between a tip of virtual scalpel and target objects, the general collision detection scheme [10] is applied. Dataset of human body structures are obtained as volumetric MRI dataset from a normal volunteer. After semiautomatic region labeling from surroundings, 3D surface of a chest wall and a heart are extracted, and then each internal region is divided into tetrahedra. Note that the authors stored basic information of tetrahedral objects such as vertices, edges and surfaces, and reduced computational cost using relational information like neighboring, parent and child pointers in overall simulation. In order to adapt real time performance and simulation quality, dynamic localization is performed using hierarchical structure of elements.

Interactive soft tissue cutting that the developed system provides is shown in Fig. 7. The chest wall has $4 \%$ tension, the elastic coefficient $100 \mathrm{~N} / \mathrm{m}$, the damper coefficient $0.05 \mathrm{Ns} / \mathrm{m}$ and the density $0.90 \mathrm{~g} / \mathrm{cm}^{3}$. Simulated volumetric cuts are smooth and zigzag cuts are reduced due to the adaptive scheme. A bright part of the chest wall on 
near the virtual scalpel means the region where physical simulation is applied. Measurement of calculation time confirms that overall algorithms are calculated within 10 msec. Force feedback architecture is also strictly managed by parallel computing and valid complementation.

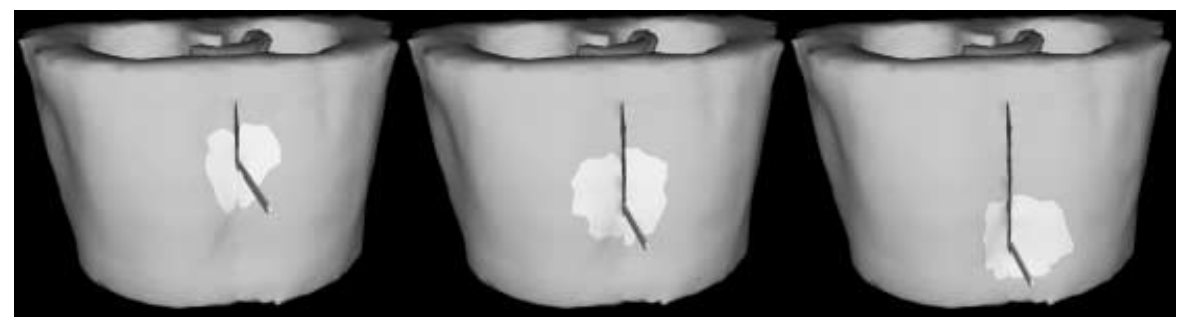

Fig. 7. Interactive soft tissue cutting on virtual chest wall (1sec, $2 \mathrm{sec}, 3 \mathrm{sec})$

Fig. 8 depicts a practical use of the framework for planning of minimally invasive surgery. A part of the myocardium appears in the back of the incised chest wall. Using this system, surgeons can discuss and learn strategies based on the reconstructed surgical field from patient's dataset. These figures demonstrate that the proposed methods can improve the applicability of interventional surgery simulators.

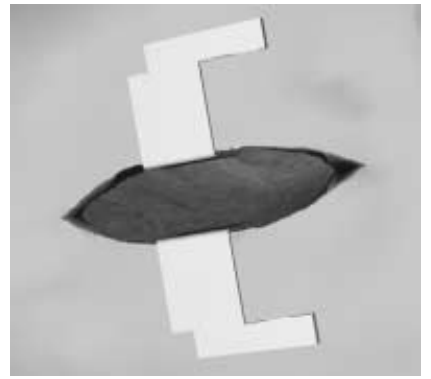

Fig. 8. Application for planning and training of minimally invasive surgery

\section{Conclusion}

In this paper, the authors presented an advanced framework that simulates soft tissue cutting, which is one of basic techniques in surgery simulation. The proposed framework is simple to implement, and integrates topological change into deformable models based on physically consistent manner. 3D MRI dataset acquired from a normal volunteer was applied to the developed system with a force feedback device. Measurement of the calculation time and demonstration of simulation quality confirmed that the model contributes to surgical planning and training. A future direction of this work is to construct a patient specific surgery simulator with tissue cutting and tearing in MIS. The authors also aim to improve surgical realism of soft tissue models such as physiological description and biomechanical deformation between multiple organs. 


\section{References}

1. Pflesser B., Leuwer R., Tiede U. Hohne, K. H.: Planning and Rehearsal of Surgical Interventions in the Volume Model. Proceedings of Medicine Meets Virtual Reality, (2000) 259-264

2. Kuhnapfel U., Cakmak H.K., Mass H.: Endoscopic Surgery Training Using Virtual Reality and Deformable Tissue Simulation. Computers and Graphics, Vol. 24. (2000) 671-682

3. M. Nakao, T. Kuroda, H. Oyama, M. Komori, T. Matsuda, T. Takahashi: Planning and training of minimally invasive surgery by integrating soft tissue cuts with surgical views reproduction, In Proceedings of Computer Assisted Radiology and Surgery, (2002)

4. C. Bruyns, K. Montgomery, S. Wildermuth: A Virtual Environment for Simulated Rat Dissection. Proceedings of Medicine Meets Virtual Reality, (2001) 75-81

5. D. Bielser, M. H. Gross: Interactive Simulation of Surgical Cuts. Proceedings of Pacific Graphics, (2000) 116-125

6. S. Cotin, D. Herve, N. Ayache: A hybrid elastic model for real-time cutting, deformations, and force feedback for surgery training and system. The Visual Computer, Vol. 16, (2000) 437-452

7. L. P. Nedel and D. Thalmann: Real time muscle deformations using mass-spring systems, Computer Graphics International. (1998) 156-165

8. C. Basdogan, Chih-Hao Ho, M. A. Srinivasan, Simulation of Tissue Cutting and Bleeding for Laparoscopic Surgery Using Auxiliary Surfaces, Proceedings of Medicine Meets Virtual Reality 8, (1999) 38-44

9. S.Z. Pirzadeh, "An Adaptive Unobstructed Grid Method by Grid Subdivision, Local Remeshing, and Grid Movement", AIAA Paper 99-3255, 1999

10. D. Ruspini, K. Kolarov, O. Khatib, The Haptic Display of Complex Graphical Environments, Proceedings of Computer Graphics, (1997) 345-352 